\title{
Influencia de factores socioculturales en la evolución alejada de pacientes con insuficiencia cardíaca.
}

\author{
Pablo Castro1, Hugo Verdejo1, Eduardo Garcés², Roberto Concepción ${ }^{3}$, Luis Sepúlveda4, \\ Fernando Lanas ${ }^{5}$, Víctor Rossel ${ }^{6}$, Silvana Llevaneras ${ }^{1}$, José Luis Vukasovic ${ }^{6}$, (En \\ representación del grupo ICARO). \\ ${ }^{1}$ Pontificia Universidad Católica de Chile, ${ }^{2}$ Hospital de Valdivia, ${ }^{3}$ Hospital Dipreca, \\ 4Universidad de Chile, ${ }^{5}$ Hospital de Temuco, ${ }^{6}$ Hospital Salvador.
}

\section{Resumen}

Objetivo: Evaluar el impacto de los factores socioculturales (SC) en las características del cuidado de la insuficiencia cardíaca (IC) y la evolución post alta en pacientes admitidos con diagnóstico de IC descompensada a hospitales del registro ICARO en el período 2006-2008.

Método: Registro prospectivo de 14 hospitales. Se incorporaron en forma consecutiva pacientes admitidos con el diagnóstico de IC descompensada entre enero 2006 y mayo 2008. La mortalidad al fin del seguimiento se determinó por la base de datos del Servicio Nacional de Registro Civil e Identificación. Se definió como terapia óptima la combinación de un betabloqueador con cualquiera de los siguientes: inhibidores de la enzima convertidora de angiotensina (IECA), antagonistas del receptor de angiotensina II (ARAII), hidralazina/isosorbide o espironolactona. Las características de los pacientes se compararon mediante t de Student o chi cuadrado según correspondía. La sobrevida se evaluó mediante Kaplan-Meier.

Resultados: Los pacientes de bajo nivel SC son de mayor edad (71 $\pm 11 \mathrm{v} / \mathrm{s} 66 \pm 15$ años respectivamente, $p<0,01)$, predomina el género femenino (52.2\% v/s $26.1 \%, p<0,01)$, y su previsión fue mayoritariamente FONASA (90\%). La etiología isquémica fue más frecuente en el estrato SC alto $(34,5$ vs. 16,6\%) y la hipertensiva en el nivel SC bajo (30,3 v/s 16,6\%). La utilización de ARA I/ fue más frecuente en el nivel SC alto con una tendencia a menor utilización de IECA, el uso de betabloqueadores espironolactona hidralazina e isosorbide amiodarona y anticoagulante fue menor en el estrato SC bajo. Al alta se observó un incremento en la utilización de terapias dirigidas a la IC pero se mantuvo un menor uso de de estas terapias en el estrato SC bajo. En los pacientes con fracción de eyección ventricular izquierda disminuida no hubo diferencias en la terapia farmacológica al egreso hospitalario en los distintos niveles SC. La terapia óptima fue de 43,7\% v/s 43,33 y 51,1\% en los niveles SC bajo, intermedio y alto, respectivamente $(p=0,91)$. Los predictores independientes de mortalidad alejada fueron: edad > de 70 años, $H R=2,17$ (1,55-3,03), un bajo nivel SC, $H R=1,57(1,17-2,09)$, una fracción de eyección < a 50\%, $H R=1,49(1,04-2,14)$ y la ausencia de una terapia óptima al alta, $H R=0,52(0,41-0,66)$. La supervivencia fue marcadamente inferior en el grupo con menor nivel SC (mediana $761 \pm 47.9 \mathrm{v} / \mathrm{s} 975 \pm 82.3$, log rank test $p=0,02$ ).

Conclusión: La población con IC y menor nivel SC y edad avanzada constituye un grupo especialmente vulnerable. Los resultados ponen en evidencia la necesidad de intervenciones destinadas a asegurar accesos igualitarios a las prestaciones de salud e implementar estrategias para mejorar la adherencia a las guías de tratamiento de la IC.

Correspondencia: Dr. Pablo Castro G.

Marcoleta 367 - Santiago, Chile

Correo Electrónico: pcastro@med.puc.cl 
P. Castro, H. Verdejo, E. Garcés, R. Concepción, L. Sepúlveda, V. Rossel, et al.

\section{Influence of social and cultural factors in the late course of patients with congestive heart failure}

Aim: to evaluate de impact of social and cultural (S-C) factors in the care and course of patients with congestive heart failure (CHF) enrolled in the ICARO study (national registry for patients with heart failure).

Methods: Patients were enrolled from 2006 through 2008. They were discharged from 14 hospitals participating in the prospectively designed ICARO study. Late mortality was obtained from the national Identification registry. Optimal medical therapy was defined as the use of a betablocker in addition to any of the following: ACE inhibitors, ARA II, combination of hydralazine and nitrates, or spironolactone. Statistical analysis included Students t test, chi square and Kaplan Meir and Log-rank testing, as appropriate.

Results: Patients with a low S-C level were older (71 \pm 11 vs $66 \pm 15$ years, $p<0.01)$, more frequently females $(52.2 \%$ vs $26.1 \%, p<0.01)$ and most of them belonged in the FONASA health insurance system $(90 \%)$. Ischemic heart disease was more prevalent in the high S-C level (34.5 vs 16.6\%) and hypertension in the low S-C level (30.3 vs 16.6\%). ARA II rather than ACE inhibitors were more commonly used in the high S-C level; ACE inhibitors, betablockers, spironolactone, hydralazine-nitrates, amiodarone and anticoagulation were less frequently used in the low S-C level. After discharge a more intensive treatment of heart failure was observed; however, this was less seen in the low S-C level. Patients with decreased left ventricular ejection fraction were similarly treated in both groups. An optimal therapy for $\mathrm{CHF}$ was used in $43.7 \%, 43.3 \%$ and $51.1 \%$ in S-C levels low, intermediate and high, respectively (NS). Independent predictors for late mortality were age>70 years (HR 2.71 (Cl 1.55-3.03), low S-C level (HR $1.57, \mathrm{Cl} 1.17$ - 2.09), EF<50\% (HR 1.49, Cl 1.04-2.14) and absence of optimal medical therapy at discharge (HR 0.52, Cl 0.41-0.66). Late survival was markedly lower in the low S-C level (median $761 \pm 47.9$ vs $975 \pm 82.3, p=0.02$ ).

Conclusion: CHF patients with a low S-C level are specially vulnerable. These results help stress the need for interventions to guarantee equal access of the population to health care and to improve adherence to CHF management guidelines.

Keywords: Heart failure, social factors, epidemiology

Recibido el 26 de diciembre de 2008. Aceptado el 8 de enero de 2009

Rev Chil Cardiol 2009; 28: 51-62

\section{Introducción}

La insuficiencia cardíaca (IC) constituye un importante problema de salud pública1-3. En países desarrollados, la prevalencia e incidencia de esta enfermedad ha aumentado en forma progresiva. Probablemente, han contribuido a este fenómeno la mayor expectativa de vida, mejores tratamientos disponibles y la mayor sobrevida a otras enfermedades cardiovasculares. La situación descrita se asocia a una elevada mortalidad, necesidad de hospitalizaciones, deterioro en la calidad de vida y consumo de recursos $1,2,4,5$. La IC constituye uno de los principales motivos de hospitalización en países desarrollados como Estados Unidos y España2,6. La práctica clínica en la IC difiere llamativamente entre países, entre áreas geográficas de un mismo país y entre diferentes subgrupos de pacientes $1,3,5$. Con frecuencia las diferencias en procedimientos diagnósticos y medidas terapéuticas dependen de las peculiaridades de cada organización de salud 7,8 . También dependen de las diferencias en las características de los pacientes incluidos en los ensayos clínicos en relación a los pacientes del mundo real o registros. Este hecho puede explicar por qué evidencias de la eficacia de los distintos fármacos expresadas en guías clínicas, no corresponden necesariamente a la práctica clínica9. 
En el año 2002 a instancias de la Sociedad Chilena de Cardiología se creó el Registro Nacional de IC (grupo ICARO), que incluye a pacientes hospitalizados por IC en centros chilenos. Desde entonces se han estudiado las características

clínicas de los pacientes ingresados, tratamiento y evolución intrahospitalaria y alejada.

Al analizar las causas de hospitalización se demostró que la falta de adherencia al tratamiento médico y la dieta, constituían las principales causas de descompensación que motivaban la hospitalización, seguidas de las infecciones y la fibrilación auricular. En este registro el $61 \%$ tenía educación básica, $17 \%$ estudios superiores y $9 \%$ era analfabeto ${ }^{10}$. Existe un reconocido impacto adverso de la deprivación sociocultural sobre la salud pública en general11. Aunque las causas que contribuyen a este mayor riesgo son multifactoriales, es importante identificar aquellos factores que puedan ser modificables mediante estrategias de prevención.

Por ejemplo, una elevada mortalidad se ha observado en síndromes coronarios agudos en estratos socioeconómicos deficientes, esto se ha atribuido a una mayor prevalencia de fenómenos ateroescleróticos, un menor acceso a atención médica y a una utilización subóptima de terapias de eficacia reconocida12-14.

En la IC la deprivación socioeconómica está asociada a un incremento de las hospitalizaciones por IC y a una mayor mortalidad, los mecanismos son inciertos pero se mencionan factores asociados a: la edad, género, presencia de co-morbilidades, mayor severidad de la enfermedad y mala adherencia al tratamiento médico15-16. Estas interrogantes son relevantes ya que la IC representa una de las principales causas de hospitalizaciones, tiene una elevada mortalidad y constituye uno de los principales costos de salud. Por otro lado, en estudios epidemiológicos se ha observado una fuerte asociación entre una pobre condición de salud y bajo nivel educacional, condición marital e inestabilidad laboral, esto parece ser más marcado en pacientes que viven en condiciones de ruralidad17. El bajo nivel sociocultural puede determinar menores ingresos, inequidad en la atención medica, dificultar la comprensión de prescripciones y representar barreras en la adherencia al tratamiento e intervenciones educativas.

Una intervención posible sería mejorar el nivel educacional y sociocultural de la población y la calidad de la salud pública a nivel nacional. Siendo esto deseable es un gran desafío, y corresponde a un objetivo sostenido y de largo plazo. En el intertanto, estimamos necesario caracterizar el perfil sociocultural de la población con IC, las características de los pacientes en los distintos estratos socioculturales y su influencia sobre eventos cardiovasculares.

El conocimiento de estos factores puede contribuir a crear estrategias orientadas a mejorar la adherencia al cumplimiento de guías clínicas, intervenciones educativas adaptadas al nivel sociocultural, comprensión de las características de la patología, guías de auto-cuidado, reconocimiento precoz de síntomas o signos de descompensación y facilitación del acceso a la atención médica. Estas intervenciones podrían tener un impacto favorable en la prevención de rehospitalizaciones, nuevos eventos cardiovasculares y mortalidad, en esta población más vulnerable.

El objetivo del presente trabajo es evaluar el impacto de los factores socioculturales en las características del cuidado de la insuficiencia cardíaca (IC) y la evolución post alta en pacientes admitidos con diagnóstico de IC descompensada a hospitales del registro ICARO en el período 2006-2008.

\section{Método}

Desde enero de 2006 a agosto de 2008, en 14 centros chilenos se recolectó información prospectiva de 968 pacientes hospitalizados por IC CF III O IV mediante una ficha electrónica específicamente diseñada para este fin. El criterio diagnóstico fue: síntomas de IC y evidencias objetivas de disfunción cardíaca objetivada en el ecocardiograma o la radiografía de tórax; en casos dudosos se consideró la respuesta al tratamiento dirigido a la IC10. Se excluyeron todos los pacientes en los cuales el motivo principal de hospitalización no fue la IC y aquellos pacientes que, estando previamente asintomáticos y $\sin$ 
P. Castro, H. Verdejo, E. Garcés, R. Concepción, L. Sepúlveda, V. Rossel, et al.

antecedente de IC, presentaron IC o shock cardiogénico secundario a un cuadro coronario agudo. Se registraron características demográficas, co-morbilidad, previsión, nivel educacional, antecedentes de hospitalizaciones previas por IC, etiología de la cardiopatía de base, factores de descompensación de la hospitalización actual, hallazgos del electrocardiograma y ecocardiograma, incluida la fracción de eyección de ventrículo izquierdo (FEVI). Se consignó el tratamiento al ingreso, alta y evolución intrahospitalaria. Las historias clínicas correspondientes, incluyendo radiografías de tórax, exámenes de laboratorio y ecocardiogramas fueron revisadas por el equipo investigador de cada centro. La mortalidad al fin del seguimiento se determinó por la base de datos del Servicio Nacional de Registro Civil e Identificación.

Se definió como terapia óptima la combinación de un betabloqueador con cualquiera de los siguientes: inhibidores de la enzima convertidora de angiotensina, antagonistas del receptor de angiotensina II, hidralazina/isosorbide o espironolactona.

Para efectos de análisis, los pacientes fueron estratificados en 3 grupos correspondientes a nivel sociocultural bajo, medio y alto en base a su nivel educacional (básica completa, media completa, con educación superior completa o incompleta), ocupación (profesional/independiente, empleado no profesional, desempleado), ruralidad (urbano/rural) y previsión (ISAPRE/Seguro de salud; Beneficiario FONASA, sin previsión). Se asignó un puntaje máximo de 3 puntos para las categorías de nivel educacional, ocupación y previsión y un puntaje máximo de 1 para pacientes de origen urbano. Los pacientes con un puntaje total de 5 o más fueron asignados al estrato de nivel sociocultural alto; los pacientes con 3 o menos puntos fueron asignados al estrato sociocultural bajo.

Análisis estadístico: Las características de los pacientes se compararon mediante ANOVA con post-hoc de Bonferroni o chi cuadrado según correspondía. La sobrevida se evaluó mediante Kaplan-Meier.

Para ponderar el impacto del factor sociocultural en la mortalidad alejada, se realizó un análisis univariado para determinar covariables significativamente asociadas a nivel sociocultural que pudiesen influir en su efecto en sobrevida. Las varia- bles identificadas fueron incorporadas a un modelo de riesgo proporcional de Cox para determinar la independencia de la asociación.

\section{Resultados}

Se incluyeron 968 pacientes correspondientes al período 2006-8. Del total 314 pacientes correspondieron al estrato sociocultural bajo, 329 al intermedio y 84 pacientes al estrato sociocultural alto.

Características de los pacientes. En la tabla 1 se muestran las características de los pacientes con IC en relación al nivel sociocultural. Al comparar los pacientes de bajo nivel sociocultural con los del estrato alto, se observa que los pacientes del primer grupo son de una mayor edad $(71 \pm 11 \mathrm{v} / \mathrm{s}$ $66 \pm 15$ años respectivamente, $p<0,01)$, predomina el género femenino $(52.2 \% \mathrm{v} / \mathrm{s} 26.1 \%$, $p<0,01)$, son de menor talla $(159 \pm 11 \mathrm{v} / \mathrm{s} 170 \pm 9 \mathrm{~cm}, \mathrm{p}<0,01)$ con una tendencia a un mayor índice de masa corporal (ns).

En relación al estado marital una menor proporción de ellos está casado y su previsión fue mayoritariamente FONASA (90\%). Respecto de las etiologías de la IC existen diferencias significativas concentrándose la etiología isquémica en el estrato sociocultural alto $(34,5 \mathrm{v} / \mathrm{s} 16,6 \%)$ y la etiología hipertensiva la más frecuente en el nivel sociocultural bajo $(30,3$ vs. $16,6 \%)$.

Previo a la descompensación de la IC los pacientes correspondientes al estrato sociocultural bajo tenían una menor CF. El antecedente de infarto fue significativamente más frecuente en el estrato sociocultural más alto $35,7 \mathrm{v} / \mathrm{s} 15,2 \%$, como también el antecedente de enfermedad vascular periférica.

El antecedente de hipertensión arterial fue menor en el estrato bajo $14,9 \mathrm{v} / \mathrm{s} 32,1 \%$. (tabla1).

\section{Forma de presentación clínica y exámenes} de laboratorio al ingreso. No existieron diferencias en la forma de presentación clínica de la IC, siendo las más frecuentes, la presencia de congestión pulmonar y visceral (tabla 2). La presión arterial sístólica y diastólica, y la frecuencia cardíaca, al ingreso fueron mayores en el estrato sociocultural bajo. 
En relación a las evaluaciones de laboratorio al ingreso la albuminemia, fue significativamente menor en el estrato sociocultural bajo $(3,4 \pm 0,6 \mathrm{v} / \mathrm{s} 3,7 \pm 0,6$ $\mathrm{mg} / \mathrm{dL}$ en los estratos intermedio y alto, $\mathrm{p}<0,01)$.

Los datos de FEVI estuvieron disponibles en el $59,2 \%$ de los pacientes. En los pacientes del estrato sociocultural bajo la FEVI (\%) fue mayor $(45,3 \pm 17 \mathrm{v} / \mathrm{s}, 41,7 \pm 17 \mathrm{v} / \mathrm{s} 38,9 \pm 19$, estratos intermedio $y$ alto respectivamente, $p<0,01)$. En el electrocardiograma el QRS fue más angosto en estrato alto $(108 \pm 29 \mathrm{v} / \mathrm{s} 119 \pm 31 \mathrm{mseg} p<0,01)$. No hubo diferencias significativas en los niveles plasmáticos de creatinina, sodio, potasio hematocrito ni ácido úrico (ver tabla2). Las causas de la descompensación que motivarón la hospitalización se presentan en la tabla 3 .

Tabla 1. Características de los pacientes con IC según nivel sociocultural.

\begin{tabular}{|c|c|c|c|c|}
\hline & $\begin{array}{c}\text { Bajo } \\
\mathrm{N}=314\end{array}$ & $\begin{array}{c}\text { Intermedio } \\
\mathrm{N}=329\end{array}$ & $\begin{array}{l}\text { Alto } \\
\mathrm{N}=84\end{array}$ & $\mathbf{P}$ \\
\hline Edad, media (DS),años & $71,15 \pm 11,8$ & $68,2 \pm 14,3$ & $66,1 \pm 15,6$ & $<0,01$ \\
\hline Sexo femenino, $\mathrm{n}(\%)$ & $164(52,2)$ & $140(42,5)$ & $22(26,1)$ & $<0,01$ \\
\hline Peso & $71,3 \pm 15,7$ & $71,5 \pm 15,2$ & $75,7 \pm 15$ & $<0,01$ \\
\hline Talla & $159,6 \pm 11,3$ & $163,9 \pm 12,4$ & $170,1 \pm 9,3$ & $<0,01$ \\
\hline IMC & $26,1 \pm 4,9$ & $25,3 \pm 5,1$ & $24,7 \pm 4,3$ & 0,06 \\
\hline Casado, $\mathrm{n}(\%)$ & $159(50,6)$ & $225(68,3)$ & $58(69)$ & $<0,01$ \\
\hline Fonasa, $\mathrm{n}(\%)$ & $284(90,4)$ & $214(65)$ & $33(39,2)$ & $<0,01$ \\
\hline Área metropolitana, n(\%) & $88(28)$ & $206(62,6)$ & $69(82,1)$ & $<0,01$ \\
\hline Etiología, n (\%) & & & & $<0,01$ \\
\hline Hipertensiva & $183(58,2)$ & $100(30,3)$ & $14(16,6)$ & \\
\hline Isquémica & $59(18,7)$ & $78(23,7)$ & $29(34,5)$ & \\
\hline Valvular & $33(10,5)$ & $61(18,5)$ & $12(14,2)$ & \\
\hline Idiopática & $8(2,0)$ & $27(8,2)$ & $6(7,1)$ & \\
\hline \multicolumn{5}{|l|}{ CF previa NHYA, $n(\%)$} \\
\hline III & $112(35,6)$ & $77(23,4)$ & $16(19)$ & $<0,01$ \\
\hline IV & $12(3,8)$ & $7(2,1)$ & $0(0)$ & \\
\hline \multicolumn{5}{|l|}{ Comorbilidades, $\mathrm{n}(\%)$} \\
\hline IAM previo & $48(15,2)$ & $68(20.6)$ & $30(35.7)$ & $<0.01$ \\
\hline Hipertensión & $47(14,9)$ & $89(27.0)$ & $27(32.1)$ & $<0.01$ \\
\hline Diabetes & $100(31,8)$ & $86(26,1)$ & $26(30,9)$ & 0,26 \\
\hline Fibrilación auricular ingreso & $(31,5)$ & $(20,1)$ & $(30,2)$ & 0,06 \\
\hline EPOC & $54(17,1)$ & $30(9,1)$ & $10(11,9)$ & 0,01 \\
\hline Enf. vascular periférica & $15(5,1)$ & $33(10,4)$ & $12(14,3)$ & 0,03 \\
\hline AVE & $27(8,5)$ & $27(8,2)$ & $10(11,9)$ & 0,57 \\
\hline Alcoholismo & $34(10,8)$ & $24(7,2)$ & $6(7,1)$ & 0,24 \\
\hline
\end{tabular}

$\mathrm{EPOC}=$ Enfermedad pulmonar obstructiva crónica, $\mathrm{IAM}=$ infarto agudo al miocardio, AVE= Accidente vascular encefálico 
P. Castro, H. Verdejo, E. Garcés, R. Concepción, L. Sepúlveda, V. Rossel, et al.

Tabla 2. Formas de presentación, exámenes de laboratorio y terapia al ingreso

\begin{tabular}{|c|c|c|c|c|}
\hline & $\begin{array}{c}\text { Bajo } \\
N=314\end{array}$ & $\begin{array}{c}\text { Intermedio } \\
\mathrm{N}=329\end{array}$ & $\begin{array}{c}\text { Alto } \\
\mathrm{N}=84\end{array}$ & $\mathbf{P}$ \\
\hline \multicolumn{5}{|c|}{ Forma de presentación } \\
\hline Congestión pulmonar & $293(76,3)$ & $310(94,2)$ & $76(90,4)$ & 0,46 \\
\hline Hipotensión & $23(7,3)$ & $18(5,4)$ & $5(5,9)$ & 0,62 \\
\hline Shock & $9(2,8)$ & $11(3,3)$ & $5(5,9)$ & 0,38 \\
\hline Congestión visceral & $74(23,5)$ & $78(23,7)$ & $20(23,8)$ & 0,99 \\
\hline \multicolumn{5}{|c|}{ Signos vitales, media, DS } \\
\hline PA sistólica, mmHg & $142,1 \pm 35$ & $137 \pm 33$ & $128,9 \pm 28.1$ & $<0,01$ \\
\hline PA diastólica, mmHg & $83,9 \pm 21,9$ & $78,1 \pm 21,2$ & $76 \pm 18,7$ & $<0,01$ \\
\hline $\mathrm{FC}, \mathrm{Lt} / \mathrm{min}$ & $95,1 \pm 26,1$ & $93,4 \pm 26.4$ & $88,5 \pm 22.6$ & 0,01 \\
\hline \multicolumn{5}{|l|}{ Exámenes laboratorio } \\
\hline Hematocrito (\%) & $38,8 \pm 6,7$ & $39,1 \pm 6.4$ & $39.4 \pm 6,9$ & 0,53 \\
\hline Creatinina, mg/dl & $1,58 \pm 1,19$ & $1,64 \pm 1,52$ & $1,61 \pm 1,79$ & 0,79 \\
\hline Sodio, mEq/l & $137.3 \pm 4,9$ & $137,2 \pm 4,9$ & $137,6 \pm 4,6$ & 0,53 \\
\hline Potasio,mEq/l & $4,3 \pm 0,8$ & $4,3 \pm 0,7$ & $4,3 \pm 0,6$ & 0,59 \\
\hline Albúmina, mg/dl & $3,4 \pm 0,6$ & $3,7 \pm 0,6$ & $3,7 \pm 0.6$ & $<0,01$ \\
\hline Ac úrico, mg/dl & $7,2 \pm 2,6$ & $6 \pm 2,5$ & $7,1 \pm 2,5$ & 0,11 \\
\hline FEVI, (\%) & $45,3 \pm 17,7$ & $41,7 \pm 17,3$ & $38,9 \pm 19,2$ & 0,03 \\
\hline \multicolumn{5}{|l|}{ Terapia Ingreso $\mathrm{n},(\%)$} \\
\hline IECA & $148(47,1)$ & $138(41,99)$ & $28(33,3)$ & 0,06 \\
\hline ARAII & $19(6,1)$ & $25(7,6 \%)$ & $12(14,3)$ & 0,04 \\
\hline Betabloqueador & $74(23,6)$ & $105(31,9)$ & $37(44)$ & $<0,01$ \\
\hline Espironolactona & $47(15)$ & $82(24, .9)$ & $21(25)$ & $<0,01$ \\
\hline Diurético & $162(51,6)$ & $194(59)$ & $45(53,6)$ & 0,16 \\
\hline Digoxina & $68(21,7)$ & $81(24,6)$ & $14(16,7)$ & 0,27 \\
\hline Hidralazina & $8(2,5)$ & $20(6,1)$ & $11(13,1)$ & $<0,01$ \\
\hline Isosorbide & $44(14)$ & $78(23,7)$ & $18(21,4)$ & $<0,01$ \\
\hline Amiodarona & $23(7,3)$ & $38(11,6)$ & $17(20,2)$ & $<0,01$ \\
\hline Anticoagulante & $65(20,7)$ & $119(36,2)$ & $40(47,6)$ & $<0,01$ \\
\hline
\end{tabular}

$\mathrm{FEVI}=$ Fracción de eyección ventricular izquierda. IECA= nhibidor enzima convertidora de angiotensina, ARA II= Antagonista angiotensina II.

Tratamiento médico al ingreso y alta. Se muestran en las tablas 2 y 4 . La utilización de antagonistas del receptor de angiotensina II (ARA II) fue más frecuente en el nivel sociocultural alto con una tendencia a menor utilización de inhibidores de la enzima convertidora de angiotensina (IECA), el uso de betabloqueadores espironolactona hidralazina e isosorbide amiodarona y anticoagulante fue menor en el estrato sociocultural bajo. Al alta se observó un incremento en la utilización de terapias dirigidas a la IC pero se mantuvo un mayor uso de ARAll respecto de IECA en el estrato alto y un menor uso de betabloqueadores espironolactona, hidralazina, amiodarona y anticoagulante oral en el estrato sociocultural bajo.

Tratamiento al egreso hospitalario en pacientes con fracción de eyección disminuida $(<50 \%)$. (Tabla 5 ). No hubo diferencias significativas en la terapia farmacológica al egreso hospitalario en los distintos niveles socioculturales. La terapia óptima: Combinación de un betabloqueador con cualquiera de los siguientes: inhibidores de la enzima convertidora de angiotensina (IECA), antagonistas del receptor de angiotensina II (ARAll), hidralazina/isosorbide o espironolactona fue de $43,7 \% \mathrm{v} / \mathrm{s} 43,33$ y $51,1 \%$ en los niveles socioculturales bajo, intermedio y alto respectivamente $(p=0,91)$. 
Tabla 3. Causas descompensantes que motiva la hospitalización y nivel sociocultural.

\begin{tabular}{lccrr}
\hline & $\begin{array}{c}\text { Bajo } \\
\mathbf{N = 3 1 4}\end{array}$ & $\begin{array}{c}\text { Intermedio } \\
\mathbf{N = 3 2 9}\end{array}$ & \multicolumn{1}{c}{$\begin{array}{c}\text { Alto } \\
\mathbf{N = 8 4}\end{array}$} & $\mathbf{P}$ \\
\hline Falta adherencia a dieta, $\mathrm{n}(\%)$ & $48(15,2)$ & $55(16,7)$ & $17(20,2)$ & 0,55 \\
Falta adherencia a meds, $\mathrm{n}(\%)$ & $25(7,9)$ & $24(7,2)$ & $11(13)$ & 0,22 \\
Fibrilación auricular & $62(19,7)$ & $68(20,6)$ & $9(10,7)$ & 0,10 \\
Progresión IC & $91(28,9)$ & $148(44,9)$ & $34(40,5)$ & $<0,01$ \\
Neumonía & $57(18,1)$ & $39(11,8)$ & $12(14,3)$ & 0,08 \\
Otras infecciones & $36(11,4)$ & $31(9,4)$ & $6(7,1)$ & 0,44 \\
SCA & $29(9,2)$ & $26(7,9)$ & $10(11,9)$ & 0,50 \\
Crisis hipertensiva & $66(21)$ & $37(11,2)$ & $3(3,6)$ & $<0,01$ \\
\hline
\end{tabular}

SCA = Síndrome coronario agudo

Tabla 4. Terapia al alta hospitalaria según nivel sociocultural.

\begin{tabular}{lcccr}
\hline & Bajo & Intermedio & Alto & P \\
\hline IECA & $189(60,2)$ & $155(47,1)$ & $36(42,9)$ & $<0,01$ \\
Ara II & $16(5,1)$ & $26(7,9)$ & $13(15,5)$ & $<0,01$ \\
Betabloqueador & $107(34,1)$ & $141(42,9)$ & $47(56)$ & $<0,01$ \\
Espironolactona & $95(30,3)$ & $125(38)$ & $40(47,6)$ & $<0,01$ \\
Diurético & $211(67,2)$ & $210(63,8)$ & $46(54,8)$ & 0,10 \\
Digoxina & $79(25,2)$ & $86(26,1)$ & $16(19)$ & 0,40 \\
Hidralazina & $21(6,7)$ & $44(13,4)$ & $16(19)$ & $<0,01$ \\
Isosorbide & $46(14,6)$ & $62(18,8)$ & $11(13,1)$ & 0,24 \\
Amiodarona & $44(14)$ & $73(22,2)$ & $19(22,6)$ & 0,02 \\
Anticoagulante & $75(23,9)$ & $123(37,4)$ & $35(41,7)$ & $<0,01$ \\
\hline
\end{tabular}

IECA= Inhibidor enzima convertidora aldosterona, ARAII: antagonista receptor angiotensina.

Tabla 5. Terapia al alta hospitalaria en pacientes con fracción de eyección disminuida y nivel sociocultural.

\begin{tabular}{lcccc} 
& $\begin{array}{c}\text { Bajo } \\
\mathbf{N = 8 7}\end{array}$ & $\begin{array}{c}\text { Intermedio } \\
\mathbf{N = 1 5 7}\end{array}$ & $\begin{array}{c}\text { Alto } \\
\mathbf{N}=\mathbf{4 7}\end{array}$ & $\mathbf{P}$ \\
\hline *Terapia óptima & $38(43,7)$ & $68(43,3)$ & $24(51,1)$ & 0,91 \\
IECA & $54(62,1)$ & $86(54,8)$ & $26(55,3)$ & 0,52 \\
ARA & $5(5,7)$ & $11(7)$ & $5(10,6)$ & 0,57 \\
Betabloqueador & $43(49,4)$ & $73(46,5)$ & $28(59,6)$ & 0,29 \\
Espironolactona & $44(50,6)$ & $85(54,1)$ & $31(66)$ & 0,22 \\
Diurético & $63(72,4)$ & $108(68,8)$ & $27(57,4)$ & 0,19 \\
Digoxina & $30(34,5)$ & $48(30,6)$ & $14(29,8)$ & 0,78 \\
Hidralazina & $8(9,2)$ & $29(18,5)$ & $10(21,3)$ & 0,09 \\
Isosorbide & $10(11,5)$ & $33(21)$ & $5(10,6)$ & 0,79 \\
Amiodarona & $15(17,2)$ & $40(25,5)$ & $11(23,4)$ & 0,33 \\
Anticoagulante & $22(25,3)$ & $57(36,3)$ & $21(44,6)$ & 0,06 \\
\hline
\end{tabular}

*Terapia óptima: Combinación de un betabloqueador con cualquiera de los siguientes: inhibidores de la enzima convertidora de angiotensina (IECA), antagonistas del receptor de angiotensina II (ARAII), hidralazina/isosorbide o espironolactona. 
P. Castro, H. Verdejo, E. Garcés, R. Concepción, L. Sepúlveda, V. Rossel, et al.

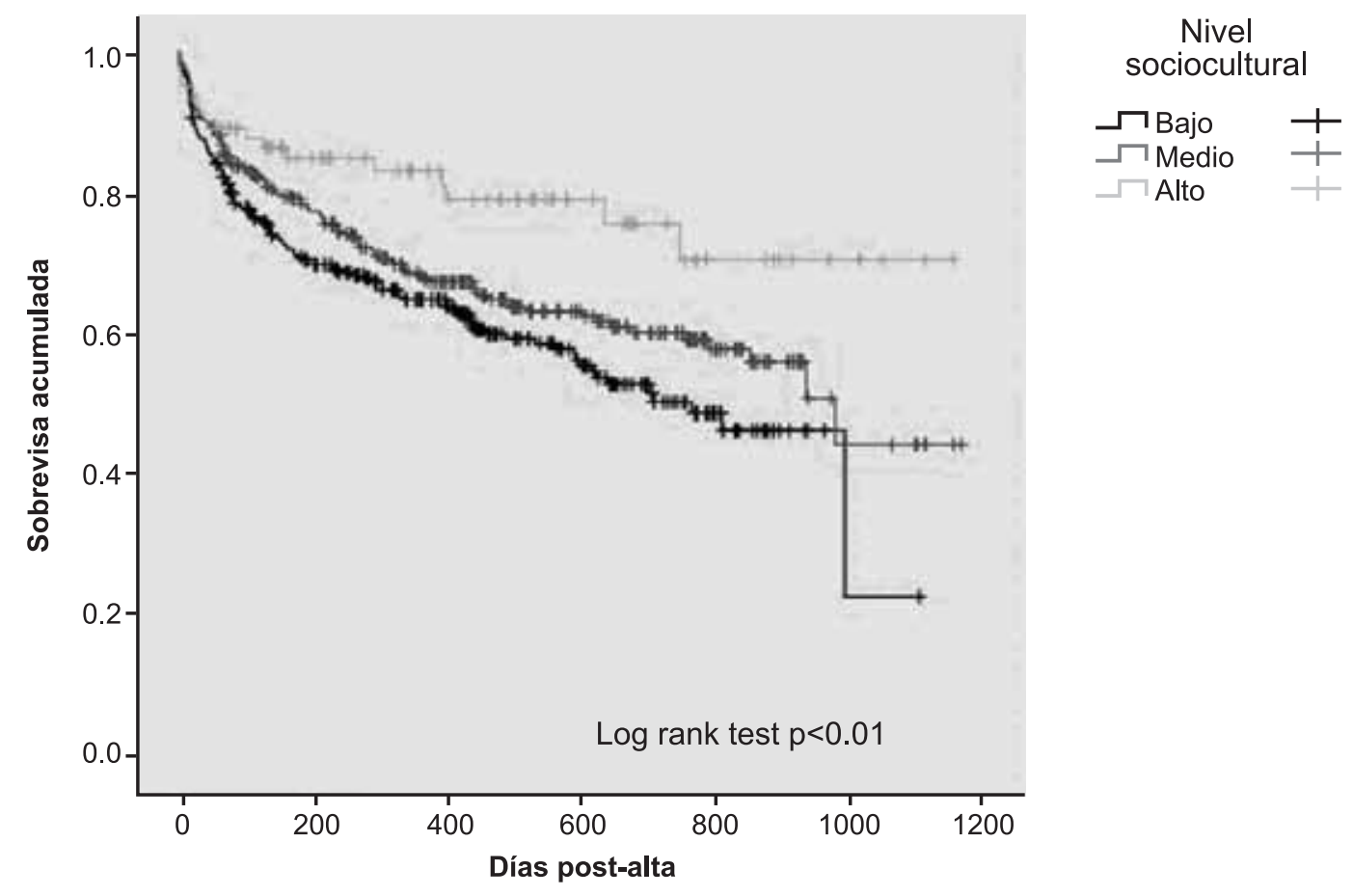

Figura 1. Curva de supervivencia en pacientes con IC egresados del hospital según nivel sociocultural.

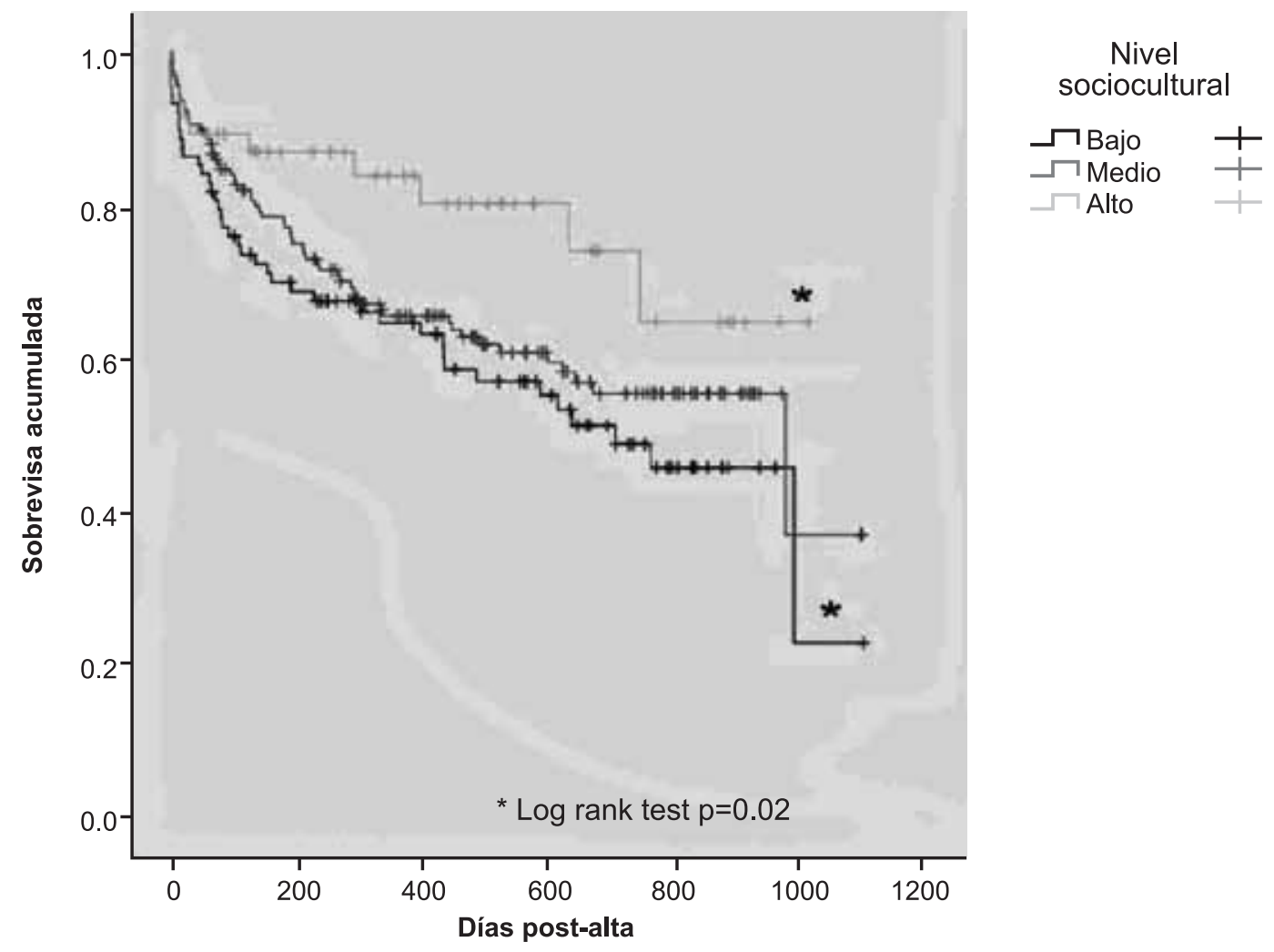

Figura 2. Curva de supervivencia en pacientes con IC con fracción de eyección disminuida post egreso según nivel sociocultural. 


\section{Discusión}

El principal hallazgo de este estudio es que el nivel sociocultural bajo junto con edad $>$ a 70 años, FEVI disminuida y la falta de una terapia farmacológica óptima al alta hospitalaria son predictores independientes de mortalidad alejada en pacientes egresados del hospital con diagnóstico de IC en el registro ICARO.

Estos resultados son relevantes porque la IC es una importante causa de morbimortalidad y aunque la sobrevida luego del diagnóstico de la IC ha mejorado en las últimas décadas la mortalidad continúa siendo elevada por lo que un mejor conocimiento de los factores que inciden en una evolución desfavorable puede contribuir a diseñar estrategias de prevención. Los pacientes con estrato sociocultural bajo son pacientes de mayor edad, fundamentalmente mujeres y con previsión FONASA. En este estrato predomina la etiología hipertensiva pese a que el antecedente de hipertensión arterial fue menos prevalente, suponemos que esto se debe a desconocimiento de esta condición. En el estrato sociocultural alto predomina la etiología isquémica en correspondencia con un mayor antecedente de infarto previo y enfermedad vascular periférica. Si bien las formas de presentación clínica fueron similares en los distintos estratos socioculturales, los pacientes correspondientes al estrato sociocultural menor tenían una menor capacidad funcional previa a la descompensación y peor estado nutricional evaluado por los niveles de albúmina al ingreso, estos hechos pueden reflejar una mayor severidad de la enfermedad, carencia nutricional y barreras ya sea a nivel del paciente como a nivel de los sistemas de atención de salud en lo que se refiere a accesos a hospitalización. En relación a la FEVI, ésta es algo mayor en el estrato sociocultural bajo, esto es concordante con una etiología a predominio hipertensiva. Sin embargo, y pese esto, los pacientes tuvieron una mayor mortalidad. Este hallazgo debe ser analizado con cautela ya que determinaciones de la FEVI no estuvieron disponibles en todos los pacientes y que además otros registros ${ }^{18}$ han mostrado un pronóstico similar en pacientes con
FEVI preservada o disminuida. Respecto de la terapia de egreso se observa en la población global una subutilización de terapias de reconocida eficacia en el manejo de la IC. Al comparar los distintos estratos socioculturales se observó que si bien los pacientes tanto del estrato sociocultural alto y bajo reciben similarmente IECA o ARAll, los pacientes del estrato bajo reciben fundamentalmente IECA que coincide con lo que esta más disponible en centros hospitalarios del Ministerio de Salud, por otro lado los ARA II, medicamentos de mayor costo y similar eficacia clínica se concentran en el estrato alto. Los pacientes del estrato sociocultural bajo reciben menos betabloqueadores, espironolactona, hidralazina, amiodarona y anticoagulante oral. Se puede especular a que los betabloqueadores que se han recomendado para la IC son menos accesibles en hospitales del sistema público. Sin embargo, como se incluyen en el grupo global pacientes con FEVI preservada y disminuida, un análisis dirigido a los pacientes con disfunción sistólica y en los cuales las recomendaciones de la terapia farmacológica esta basada en una mayor evidencia proveniente de ensayos clínicos randomizados, resulta más apropiado. De tal modo que, cuando se restringe el análisis al grupo con FEVI disminuida $(<50 \%)$, se encontró que la terapia farmacológica de la IC no es diferente en los distintos estratos socioculturales; por otro lado, cuando se consideró una terapia de calidad óptima, no existió diferencias entre los grupos, siendo en todos ellos insuficiente respecto de las recomendaciones actuales. De esto se desprende que, aún a nivel hospitalario, la prescripción de medicamentos es inadecuada. Pese a esto, un análisis de la evolución de la terapia farmacológica entre los años 2002 al 2008 muestra que han existido mejorías en la calidad de la misma (resumen Congreso Chileno Cardiología 2008, Garcés y cols, grupo ICARO). Las reciente difusión de las guías clínicas del tratamiento de la IC patrocinadas por la Sociedad Chilena de Cardiología pudiesen contribuir a una mejoría adicional ${ }^{19}$.

A nivel internacional existe escasa información de la influencia de factores socioculturales en la IC siendo inexistente información o publicaciones a nivel nacional. Esto, probablemente por la existencia 
de factores confundentes asociados. Por ejemplo, un estudio sueco encontró que un bajo nivel ocupacional era un predictor de IC20. En otro estudio (First Nacional Health and Nutricional Examination Study, NHANES), se demostró un incremento del riesgo de IC a menor nivel educacional21. Sin embargo, en otro reporte no se encontró esta asociación 22 . Estos resultados disímiles pueden estar influidos por pequeño número de pacientes incluidos, y otras variables no controladas dependientes del país o área en donde se realizó la investigación. En un reporte de Lee RJ y cols que incluyó a una cohorte con un número significativo de pacientes $(n=2157)$ de sexo femenino el nivel educacional fue un predictor independiente de mortalidad cardiovascular23.

En Chile, no existe información de la influencia del nivel educacional en la IC, en relación a esto se pueden postular varias razones que expliquen la asociación de un bajo nivel educacional y riesgo de enfermedades cardiovasculares. Entre estas se señalan factores genéticos, grado de exposición a infecciones, acceso a salud, estrés, ausencia de redes sociales y otros como consumo de alcohol, tabaco y malnutrición 24 .

Intervenciones educativas especialmente adaptadas a determinados estratos especialmente vulnerables pueden ser efectivas, es así como en una población indigente, la evolución de la IC fue favorablemente modificada por intervención educativa dirigida especialmente a este estrato poblacional 25 .

En otro estudio realizado en una cohorte brasilera de 494 pacientes ingresados por IC los factores socio-económicos estuvieron asociados a una mayor mortalidad alejada. Este pronóstico desfavorable se asoció a dificultades en el acceso y a una menor calidad de la atención de salud pública (26).

En la primera publicación de nuestro grupo encontramos que la mayoría de los pacientes que se ingresan por IC correspondió a pacientes con IC crónica descompensada. Las principal causa de descompensación fueron la mala adherencia a la dieta y a los medicamentos (30\% de los casos), seguida de las infecciones y la fibrilación auricular ${ }^{10}$.
Una elevada proporción de pacientes tenía un bajo nivel educacional. De esta observación surgió estudiar la posible influencia de factores socioculturales en la que se incluye esta variable en una cohorte mejor caracterizada y que corresponde al presente reporte que incluyó sólo a pacientes entre los años 2006 al 2008, período en el cual se contó con una ficha electrónica de recolección de información accesible por Internet.

Fortalezas y limitaciones del presente estudio: Entre las fortalezas de este estudio se encuentra que entrega la primera información nacional disponible en relación a la influencia de factores socioculturales en la evolución de pacientes con IC, un problema de salud creciente y relevante. Se incluyó un considerable número de pacientes y de distintos centros, distribuidos por todo el país, la información se recolectó en forma prospectiva y se incluyeron pacientes con fracción de eyección preservada y disminuida. Además, el seguimiento fue de un $100 \%$ y se consideró un endpoint duro como mortalidad global.

En relación a las limitaciones de nuestro estudio se incluyen que este es un registro voluntario, por consiguiente, existen casos no incluidos; también existen posibles dificultades en la adjudicación de la etiología de base de la cardiopatía, ausencia de determinación de fracción de eyección en todos los pacientes y ausencia de información respecto al ingreso económico. Si bien se incluyó en el análisis la calidad de de la terapia recibida al momento del egreso, desconocemos la adherencia al tratamiento en el seguimiento. Finalmente pueden existir factores no evaluados como posibilidad de acceso a centros de atención de salud y dificultades en la comprensión entre otros. Otro aspecto a considerar es que se utilizó la mortalidad global y no la cardiovascular, sin embargo, en estudios epidemiológicos y en la información del registro de identificación la mortalidad global parece ser un elemento más objetivo e identificable de evaluación. Por último, los resultados de un seguimiento telefónico de los pacientes ingresados al registro ICARO son aún preliminares y no concluyentes. Para contestar estas interrogantes, se encuentra en desarrollo un 
proyecto FONIS, en el que participan algunos centros del grupo ICARO, en los cuales se está efectuando una intervención educativa y de seguimiento adaptada a cada estrato educacional. Se enfatiza el autocuidado, la adherencia a la terapia, reconocimiento precoz de síntomas o signos de descompensación, e intervenciones de enfermería y derivación médica en los casos necesarios. Para estos efectos, el proyecto entrega un teléfono celular para establecer contacto con el paciente en forma protocolizada.

\section{Conclusiones}

La población con IC y menor nivel sociocultural y de edad avanzada constituye un grupo especialmente vulnerable. Los resultados ponen en evidencia la necesidad de intervenciones destinadas a asegurar accesos igualitarios a cuidados y prestaciones de salud. Junto con esto es perentorio a nivel de los centros hospitalarios intervenciones destinadas a mejorar la adherencia a las guías de tratamiento de la IC.

\section{Referencias}

1. Heart Failure: evaluation and care of patients with left ventricular dysfunction. US Department of Health and Human Services. Publication No 94-0612, Maryland, 1994.

2. RODRÍGUEZ ARTALEJO F, GUALLAR-CASTILLÓN P, BANEGAS R, DEL REY J. Trends in hospitalization and mortality for heart failure in Spain. Eur Heart J 1997; 18: 1537-40.

3. SHARPE N. Management principles: much more to be gained. En: Heart failure management. London: Martin Dunitz, 2000.

4. ESC Guidelines for the diagnosis and treatment of acute and chronic heart failure 2008 The Task Force for the Diagnosis and Treatment of Acute and Chronic Heart Failure 2008 of the European Society of Cardiology. Eur J Heart Fail. 2008 Oct; 10: 933-89.

5. HOBBS FDR, DAVIS RC, MCLEOD S, MARSHALL T, KENKRE J, LANCASHIRE R. Prevalence of Heart Failure in high risk groups. J Am Coll Cardiol 1998; 31: 5-85.

6. ERHARDT L, CLINE C. Community Management of Heart Failure 2000. En: Heart failure management. London: Martin Dunitz, 2000.

7. COHEN-SOLAL A, DESNOS M, DELAHAYE F, EMERIAN JP,HANANIA G. A national survey of heart failure in French hospitals. Eur Heart J 2000; 21: 763-9.

8. COWIE MR, MOSTERD A, WOOD DA, DECKERS JW, POOLE-WILSON PA, SUTTON GC, et al. The epidemiology of heart failure. Eur Heart J 1997; 8: 208-25.

9. PHILBIN EF, ANDREOU C, ROCCO TA, LYNCH LJ, BAKER SL. Patterns of angiotensin-converting enzyme inhibitor use in congestive heart failure in two community hospitals. Am J Cardiol 1996; 77: 832-8.

10. CASTRO P VUKASOVIC JL, GARCÉS E, SEPÚLVEDA L, FERRADA M, ALVARADO $S$. Insuficiencia cardíaca en hospitales chilenos: resultados del Registro Nacional de Insuficiencia Cardíaca, Grupo ICARO. Rev Méd Chile 2004; 132: 655-662

11. MCALISTER FA, MURPHY NF, SIMPSON CR, STEWART S,
MACINTYRE K, KIRKPATRICK M, et al. Influence of socioeconomic deprivation on the primary care burden and treatment of patients with a diagnosis of heart failure in general practice in Scotland: population based study BMJ 2004; 328; 1110.

12. ALTER DA, NAYLOR CD, AUSTIN P, TU JV. Effects of socioeconomic status on access to invasive cardiac procedures and on mortality after acute myocardial infarction. N Engl JMed 1999; 341: 1359-67.

13. KAPLAN GA, KEIL JE. Socioeconomic factors and cardiovascular disease: a review of the literature. Circulation 1993; 88: 1973-98.

14. MACLEOD MC, FINLAYSON AR, PELL JP, FINDLAY IN. Geographic, demographic, andsocio-economic variations in the investigation and management of coronary heart disease in Scotland. Heart 1999; 81: 252-6.

15. STRUTHERS AD, ANDERSON G, DONNAN PT, MACDONALD T. Social deprivation increases cardiac hospitalisations in chronic heart failure independent of disease severity and diuretic non-adherence. Heart 2000; 83: 12-6.

16. MACINTYRE K, CAPEWELL S, STEWART S, CHALMERS J, BOYD J, FINLAYSON A. Evidence of improving prognosis in heart failure. Trends in case fatality in 66547 patients hospitalized between 1986 and 1995. Circulation 2000; 102: 1126-31.

17. GOLDMAN LE, DUDLEY RA. United States rural hospital quality in the Hospital Compare database-accounting for hospital characteristics. Health Policy 2008; 87: 112-27.

18. VUKASOVIC JL, CASTRO P, SEPÚLVEDA L, NAZZAL C, GARCÉS E, CONCEPCIÓN R, et al. Características de la insuficiencia cardiaca con fracción de eyección preservada: resultados del registro Chileno de insuficiencia cardiaca, ICARO. Rev Med Chile. 2006; 34: 539-48.

19. ALFARO M, CASTRO P, JALIL J, LLANCAQUEO M, SEPÚLVEDA L, VUKASOVIC JL. Guías chilenas para 
P. Castro, H. Verdejo, E. Garcés, R. Concepción, L. Sepúlveda, V. Rossel, et al.

el tratamiento de la insuficiencia cardiaca. Rev Chil Cardiol 2007; 26: 473-504.

20. ERIKSSON H, SVARDSUDD K, LARSSON B, OHLSON LO, TIBBLIN G, WELIN L. Risk factors for heart failure in the general population: the study of men born in 1913. Eur Heart J 1989; 10: 647-e56.

21. HE J, OGDEN LG, BAZZANO LA, VUPPUTURI S, LORIA C, WHELTON PK. Risk factors for congestive heart failure in US men and women:NHANES I epidemiologic follow-up study. Arch Intern Med 2001; 161: 996-1002.

22. CHEN YT, VACCARINO V, WILLIAMS CS, BUTLER J, BERKMAN LF, KRUMHOLZ HM. Risk factors for heart failure in the elderly: a prospective community-based study. Am J Med 1999; 106: 605-12.
23. LEE RJ, PAULTRE F, MOSCA L. The association between educational level and risk of cardiovascular disease fatality among womwn with cardiovascular disease. Women's Health Issues 2005; 15: 80-88.

24. STEPTOE A, MARMOT M. The role of psychobiological pathways in socio-economic inequalities in cardiovascular disease risk. Eur Heart J 2002; 23: 13-25.

25. O'CONNEL AM, CRAWFORD MH, ABRAMS J. Heart failure disease management in an indigent population. Am Heart $\mathrm{J}$ 2001; 141: 254-8.

26. DE CAMPOS C, YAMADA A, ARAUJO F, PEREIRA A, MANSUR A. Socioeconomic factors in the prognosis of heart failure in a Brazilian cohort. International Journal of Cardiology 2006; 113: 181-187 